\title{
Preliminary results of a new intercalary modular endoprosthesis for the management of diaphyseal bone metastases
}

\author{
Kadir Büyükdoğan, $M^{1}{ }^{1}$, Barlas Göker, $\mathrm{MD}^{2}\left(\mathbb{D}\right.$, Mazhar Tokgözoğlu, MD²D, Ulukan İnan, $\mathrm{MD}^{3}(\mathbb{D}$,

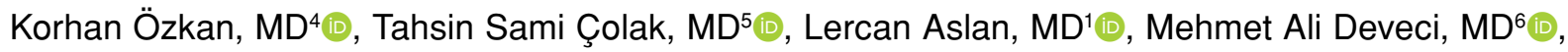 \\ Mehmet Ayvaz, $\mathrm{MD}^{2}$ (D)
}

\begin{abstract}
'Department of Orthopedics and Traumatology, Koç University Hospital, Istanbul, Turkey
${ }^{2}$ Department of Orthopedics and Traumatology, Hacettepe University, Faculty of Medicine, Ankara, Turkey

${ }^{3}$ Department of Orthopedics and Traumatology, Osmangazi University, Faculty of Medicine, Eskişehir, Turkey

${ }^{4}$ Department of Orthopedics and Traumatology, Medeniyet University, Faculty of Medicine, Istanbul, Turkey

${ }^{5}$ Department of Orthopedics and Traumatology, Necmettin Erbakan University, Faculty of Medicine, Konya, Turkey

${ }^{6}$ Department of Orthopedics and Traumatology, Koç University, Faculty of Medicine, Istanbul, Turkey
\end{abstract}

Treatment of skeletal metastases is a significant part of cancer care. The prevalence of metastatic bone lesions are expected to increase annually, as the recent advances in medical management of cancer patients result in prolonged survival. ${ }^{[1]}$ Bone after the lung and liver is the third most common site of metastasis in cancer patients and skeletal-related events, as bone metastases considerably affect activities of daily living and quality of life in cancer patients ${ }^{[2]}$ Multiple options for the treatment of long bone diaphyseal metastases have been defined including diaphyseal

Received: March 08, 2021

Accepted: May 17, 2021

Published online: November 19, 2021

Correspondence: Mehmet Ayvaz, MD. Hacettepe Üniversitesi Tıp Fakültesi Ortopedi ve Travmatoloji Anabilim Dalı,

06230 Altındağ, Ankara, Türkiye.

E-mail: mehmetayvaz@gmail.com

Doi: $10.52312 /$ jdrs.2021.51

Citation: Büyükdoğan K, Göker B, Tokgözoğlu M, İnan U, Özkan $K$, Çolak TS, et al. Preliminary results of a new intercalary modular endoprosthesis for the management of diaphyseal bone metastases. Jt Dis Relat Surg 2021;32(3):713-720.

(C2021 All right reserved by the Turkish Joint Diseases Foundation

This is an open access article under the terms of the Creative Commons Attribution-NonCommercial License, which permits use, distribution and reproduction in any medium, provided the original work is properly cited and is not used for commercial purposes (http://creativecommons.org/licenses/by-nc/4.0/).

\section{ABSTRACT}

Objectives: This study aims to evaluate functional outcomes of patients and to analyze complication rates of modular intercalary endoprosthetic reconstruction after resection of metastatic diaphyseal bone lesions.

Patients and methods: Between December 2017 and February 2020, 22 patients (15 males, 7 females; median age: 64.2 years; range, 49 to 91 ) who underwent reconstruction with modular intercalary endoprostheses for metastatic bone tumors at five different centers were retrospectively analyzed. Age, sex, diagnosis, follow-up duration, previous treatments of patients, and resection lengths were recorded. The Musculoskeletal Tumor Society Scores (MSTS) were used to assess functional status of available patients at the final follow-up. Failures were categorized according to the Henderson classification.

Results: Locations of the resected tumors included 10 humeri (45.5\%), five tibiae $(22.7 \%)$, and seven femurs (31.8\%). The length of the resected tissues ranged from $35 \mathrm{~mm}$ to $180 \mathrm{~mm}$. Seven patients $(31.8 \%)$ died of disease, and one patient died of pneumonia within follow-up period. The functional outcomes of surviving patients were satisfying with a median MSTS score of $86.9 \%$ (range, 70 to $100 \%$ ) at a median follow-up of 17 (range, 8 to 26) months. There were two cases of type II (9\%), one cases of type IIIa (4.5\%), two cases of type IIIb (9\%), and one case of type IV (4.5\%) failure. Complications were most commonly observed in tibial reconstructions.

Conclusion: The good short-term functional results were achieved in surviving patients. Uncomplicated patients were able to perform daily living activities without limitations. The overall rate of complications was relatively low and, among them, mechanical problems were the most commonly encountered problems.

Keywords: Diaphyseal bone metastases, endoprosthesis, intercalary modular endoprosthesis, modular tumor prosthesis. 
reconstructions with intramedullary nailing (IMN) and endoprosthetic reconstruction with intercalary prostheses. ${ }^{[3]}$

To determine the optimal treatment, the surgeon should first consider the general condition and expected survival of the patient, along with the remaining healthy bone stock. ${ }^{[3]}$ Patients with better prognoses and relatively longer life expectancies may better tolerate and benefit more from aggressive, yet durable treatment methods. ${ }^{[4]}$ In the literature, modular intercalary endoprosthesis have been used with indications such as the presence of extensively lytic lesions that hinders IMN reconstruction, metastases that spare the metaphysis and epiphyses of long bones, potentially increased survival with wide resection of the tumor, particularly in case of a solitary metastasis and intent of early weight-bearing and mobilization. ${ }^{[4,5]}$ Modularity allows the surgeon to restore limb length intraoperatively by customizing the implant size accordingly. Encouraging patients to return to a certain activity level sooner may provide a significant benefit in the functional outcomes of metastatic cancer patients who have a potentially shorter lifespan. However, these implants have an inherent risk of complications including infection, structural failure, and recurrence. ${ }^{[5]}$ The literature is scarce regarding the functional outcomes and complications of intercalary endoprostheses.

In the present study, we aimed to evaluate functional outcomes of patients and to analyze complication rates of modular intercalary endoprosthetic reconstruction after resection of metastatic diaphyseal bone lesions.

\section{PATIENTS AND METHODS}

This multi-center, retrospective study was conducted at Hacettepe University, Faculty of Medicine, Department of Orthopedics and Traumatology between January 2017 and June 2020. A total of 22 patients (15 males, 7 females; median age: 64.2 years; range, 49 to 91 years) who underwent reconstruction with modular intercalary endoprostheses (ESTAS Medical, Sivas, Turkey) for diaphyseal bone defect after resection of bone metastasis at five different centers were included. Primary bone tumors, pediatric patients, and tumors which did not include the diaphyses were excluded. Data including age, sex, diagnosis, follow-up duration, previous treatments, and resection lengths were obtained from patient charts. The functional status of the patients at the final follow-up and complications during follow-up were recorded. A written informed consent was obtained from each patient. The study protocol was approved by the Hacettepe University Ethics Committee (No: 2020/13-19, Date: 25/08/2020). The study was conducted in accordance with the principles of the Declaration of Helsinki.

Open or closed biopsy techniques were used to confirm the histological diagnosis prior to surgery

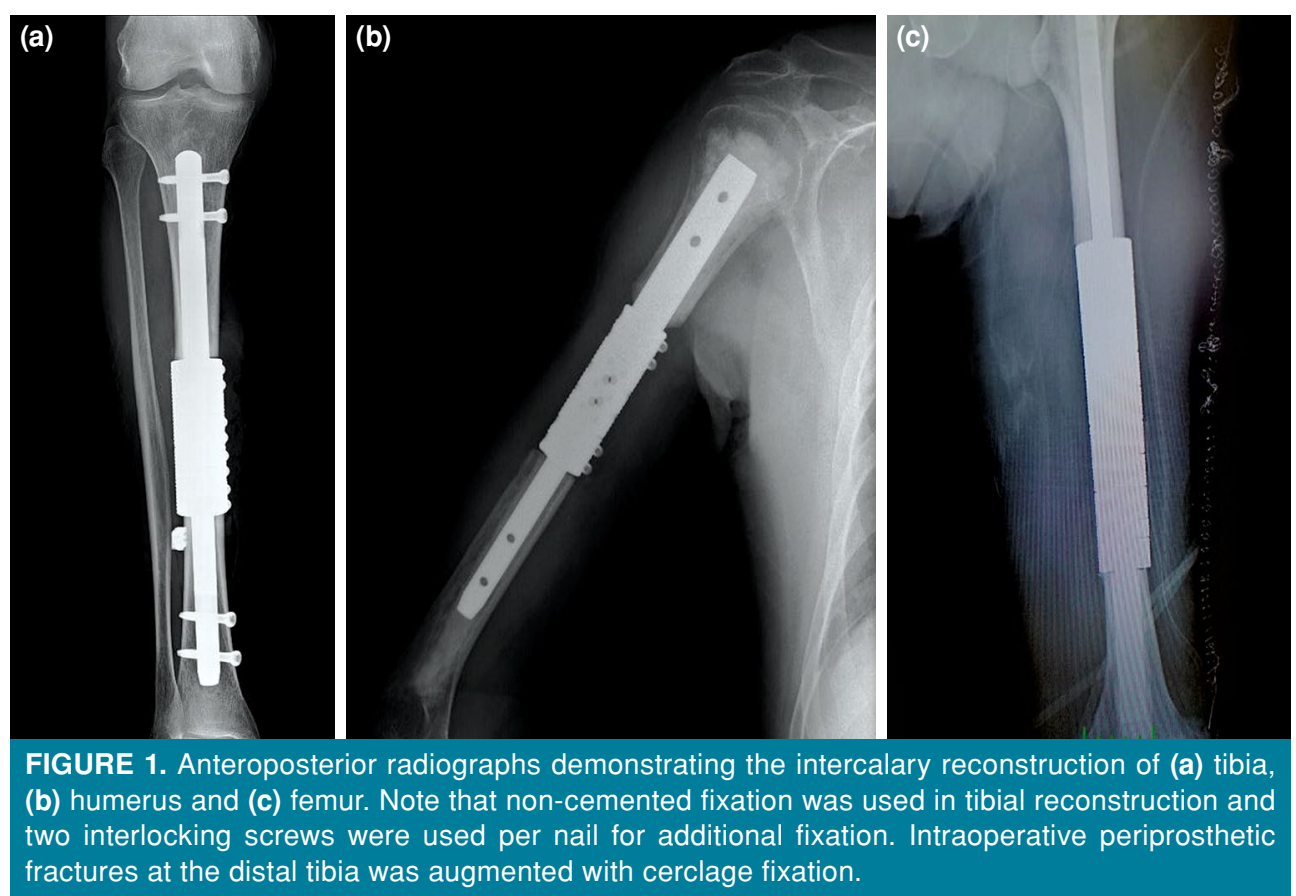


for all patients. Preoperative chemotherapy and/or radiotherapy were scheduled based on the diagnosis and extent of the tumor. The indication for the use of this endoprosthesis was segmental bone loss due to bone metastases without involving the joints above and below. The patients were considered fit for the reconstruction with the ESTAS modular intercalary endoprosthesis (ESTAS Eksantrik Sanayi ve Ticaret A.Ş, Sivas, Turkey), in the setting of skeletal defect size more than $35 \mathrm{~mm}$ after en bloc resection of tumors, and subsequently achieving a bone stock more than $50 \mathrm{~mm}$ for femur and tibia and more than $30 \mathrm{~mm}$ for humerus at each end of the native bone (Figure 1). This implant is the equivalent medical device of OsteoBridge ${ }^{\mathrm{TM}}$ IDSF (Merete $\mathrm{GmbH}$, Berlin, Germany), but differs from the original design by an improved clamp mechanism. The implant is CE-certificated (M.2021.106.14229), and all components of the system are manufactured from Ti6A14V ELI alloy (ASTM F136, ISO 5832-3). In brief, the implant design consists of two intramedullary nails at the edges with a spacer in between. The spacers are semi-circular shells that are connected with $3.5-\mathrm{mm}$ or $5-\mathrm{mm}$ locking screws, and multiple spacers can be combined for larger defects $(>65 \mathrm{~mm})$ (Figure 2). Surface treatment applied to stems include grit blasting with abrasive aluminum-oxide (A12O3) sands to increase the surface area and passivating the surface by sequential anodization to form a controlled titanium dioxide $\left(\mathrm{TiO}_{2}\right)$ layer on stems. This process increases the physical hydrophilicity of the surface and achieves the roughness that allows appositional bone growth on stem. Stems were implanted into native bone with or without polymethylmethacrylate (PMMA) cement. In the setting of non-cemented fixation, two interlocking screws were used per nail for additional fixation. In this case series, cemented fixation was the preferred method of fixation for the majority of the cases. After cement application into the canals, the nails were inserted. An appropriate size spacer was selected to fill the defect, and the shells were fixed around the (a)

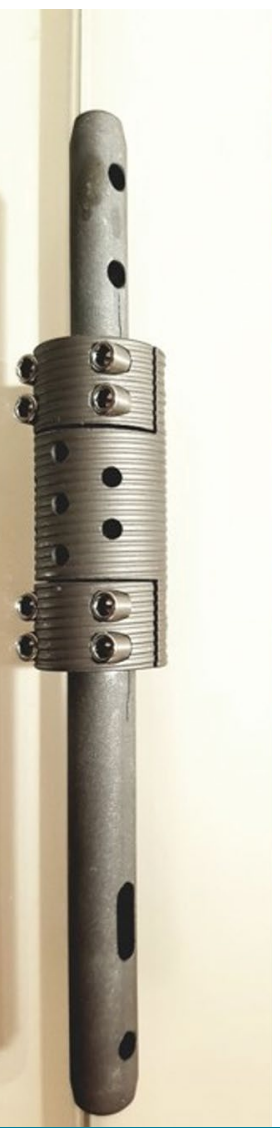

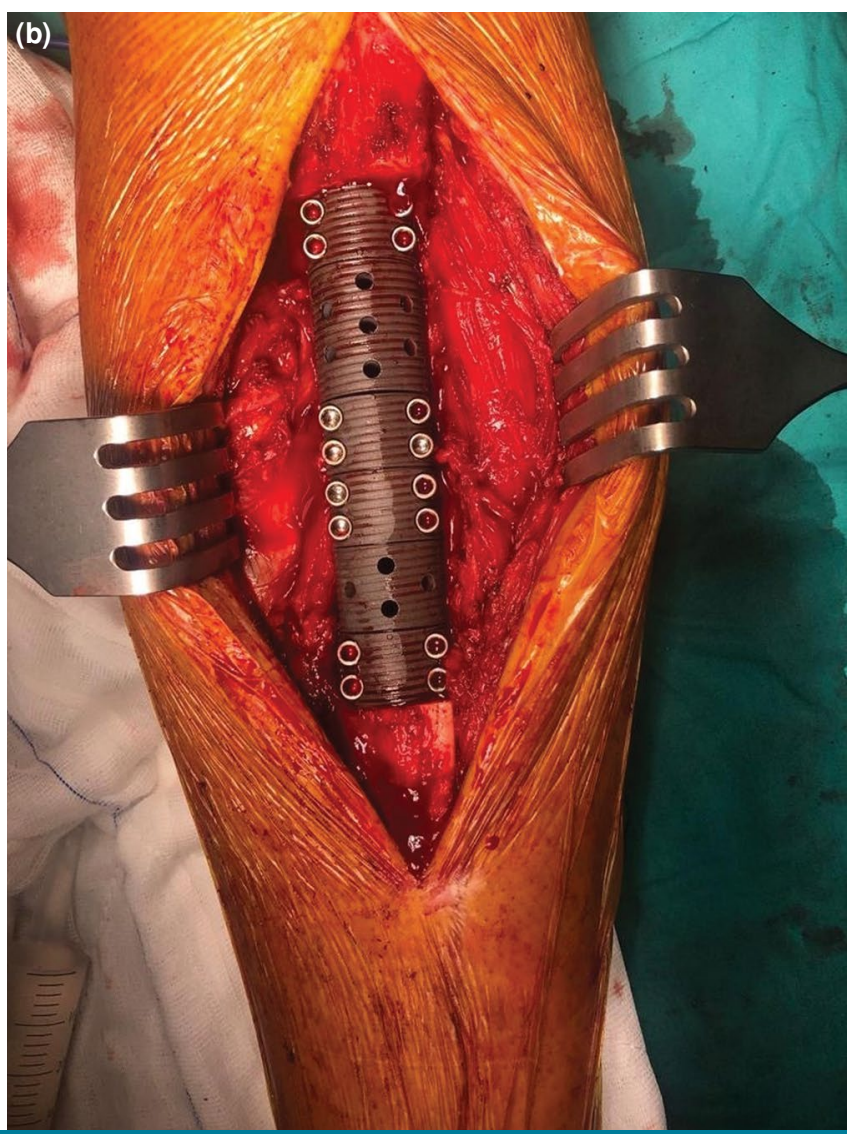

FIGURE 2. (a) Implant consists of two intramedullary nails at the edges with a spacer in between. The spacers are semi-circular shells that are connected with $3.5-\mathrm{mm}$ or $5-\mathrm{mm}$ locking screws, and multiple spacers can be combined for larger defects. (b) Intraoperative image of a modular prosthesis implanted after tibia diaphysis resection. 


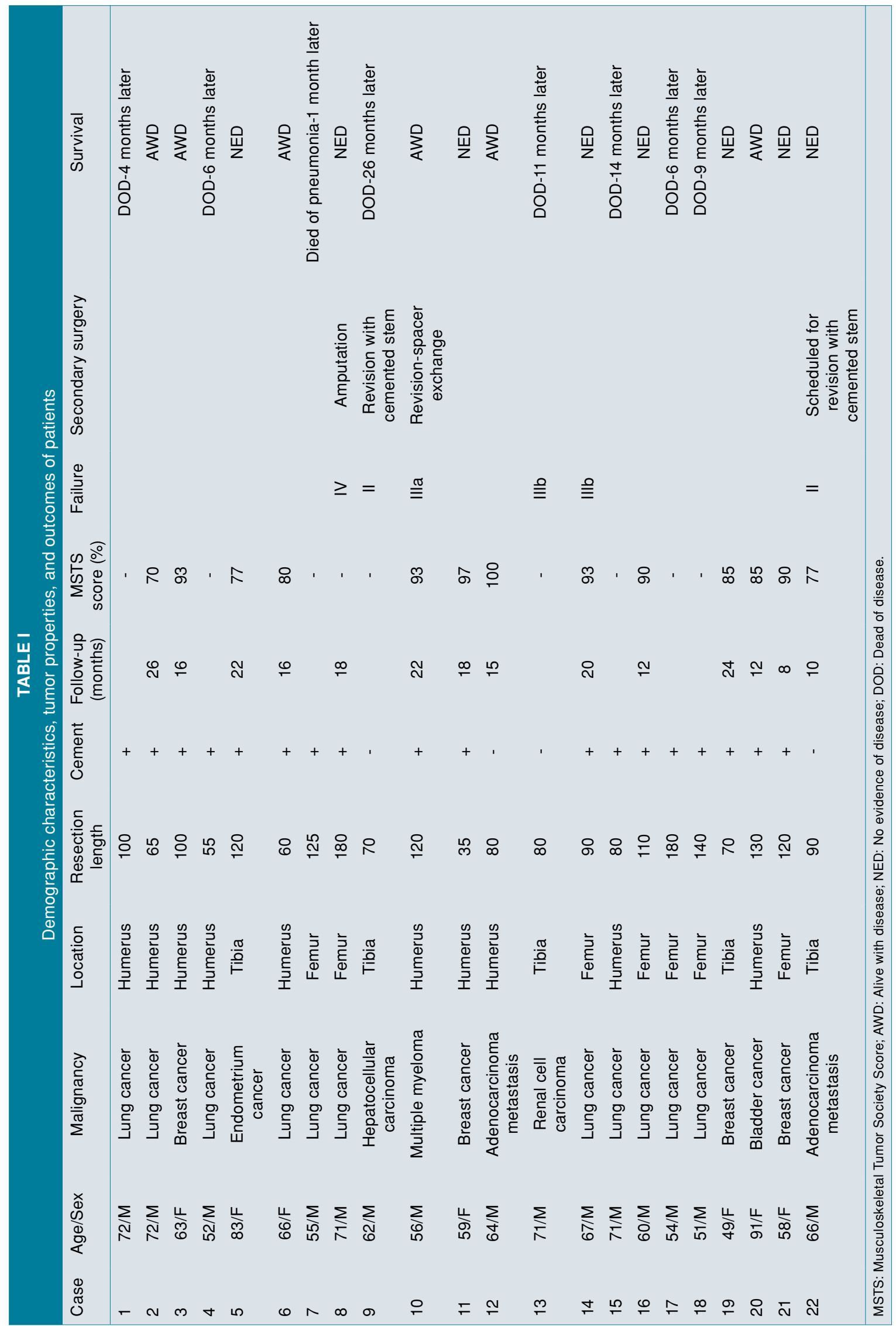


nails with eight screws per spacer unit after ensuring the rotational alignment.

Functional evaluation of the patients was performed using the Musculoskeletal Tumor Society Score (MSTS) ${ }^{[6]}$ The system assigns numerical values from 0 (significant impairment) to 5 (a full function without any impairment) for each of six categories: pain, function, and emotional acceptance as general criteria; supports, walking ability, and gait for lower extremity-specific criteria; hand supports, manual dexterity, and lifting ability for upper extremity-specific criteria. A maximum of 30 points can be achieved without any impairment, and all values are expressed in percentages.

The complications were classified according to the system proposed by Henderson et al. ${ }^{[7]}$ According to this classification system, soft tissue failures were categorized as type I, aseptic loosening as type II, structural failures as type III (IIIa, implant breakage; IIIb, periprosthetic osseous fracture), infection as type IV and tumor progression as type V. Type I, II and III failures were subsequently grouped as mechanical, type IV, and V failures were classified as non-mechanical complications.

\section{Statistical analysis}

Statistical analysis was performed using the IBM SPSS version 26.0 software (IBM Corp., Armonk, NY, USA). Continuous variables were expressed in median (min-max), while categorical variables were expressed in number and frequency. The Fisher's exact test was used to analyze the association between complication rates and anatomic sites. Due to the non-normally distributed data set, the Spearman correlation was used to analyze the association between MSTS scores and resection length. A two-tailed $p$ value of $<0.05$ was considered statistically significant.

\section{RESULTS}

Of the patients, $90.9 \%$ received adjuvant chemotherapy and $72.7 \%$ received radiotherapy. The most common presenting symptom was pain $(100 \%)$ in all patients and three patients $(13.6 \%)$ presented with pathological fractures. The locations of the resected tumors included 10 humeri $(45.5 \%)$, five tibiae (22.7\%), and seven femurs (31.8\%) (Table I). The median length of the resection was 100 (range, 35 to 180$) \mathrm{mm}$. The median proximal stem length was 61 (range, 50 to 70 ) $\mathrm{mm}$, and the median distal stem length was 72 (range, 50 to 100) $\mathrm{mm}$. The PMMA bone cement was used for stem fixation in 18 patients $(81.8 \%)$.
During the follow-up period, seven patients $(31.8 \%)$ died of disease at a median survival of 8.6 (range, 4 to 26) months. One patient died of pneumonia one month after the operation, and after including this patient, the mortality rate increased to $36.3 \%$. Eight patients $(36.3 \%)$ had no evidence of disease, and six patients $(27.2 \%)$ were alive with disease. The median MSTS scores of surviving patients were $88.2 \%$ (range, 70 to $100 \%$ ) for upper extremity, and $85.3 \%$ (range, 77 to 93\%) for lower extremity at a median follow-up of 17 (range, 8 to 26) months. When both upper and lower extremities were combined, the mean MSTS score was found to be $86.9 \%$ (range, 70 to $100 \%$ ). For most of the surviving patients, functional outcomes were satisfying. All uncomplicated patients were able to bear full weight and perform daily living activities without limitations. The results of the Spearman correlation revealed no significant relationship between the resection length and MSTS scores $(\mathrm{r}=0.15, \mathrm{p}=0.627)$.

There were two cases of type II (9\%), one cases of type IIIa (4.5\%), two cases of type IIIb (9\%), and one case of type IV (4.5\%) failure, according to the classification system based on Henderson et al. ${ }^{[7]}$ (Table II). Aseptic loosening was observed in two patients $(9 \%)$ who received cementless stem fixation after tibial resections. One patient was revised with a cemented stem at 12 months after index surgery. Another patient was scheduled for revision surgery with cemented tibial stem at nine months after index surgery, but did not undergo revision by the time of writing of the manuscript. Failure of spacer locking screws was seen in one patient's $(4.5 \%)$ humerus at 12 months after humeral resection, and this case was revised with spacer exchange that allows fixation with 5-mm locking screws. Two patients $(8.3 \%)$ had periprosthetic fractures at the proximal femur and distal tibia intraoperatively and were augmented with cerclage fixation. One patient $(4.5 \%)$ with femur reconstruction had an infection and was treated with

\section{TABLE II}

Complications classified according to Henderson et al. ${ }^{[7}$

\begin{tabular}{lcc} 
Failure type & $\mathrm{n}$ & $\%$ \\
\hline Type I (soft tissue failure) & 0 & 0 \\
Type II (aseptic loosening) & 2 & 9 \\
Type III (structural failures) & 3 & 13.5 \\
$\quad$ Type IIIa (implant breakage) & 1 & 4.5 \\
Type IIIb (periprosthetic fracture) & 2 & 9 \\
Type IV (infection) & 1 & 4.5 \\
Type V (tumor progression) & 0 & 0
\end{tabular}


debridement and a course of intravenous antibiotics, but underwent amputation due to uncontrolled infection. Mechanical, non-mechanical, and overall complication rates were calculated as $22.7 \%, 4.5 \%$, and $27.2 \%$, respectively. Totally, $60 \%(3 / 5)$ of the mechanical complications and 50\% (3 of 6) of the overall complications were observed in the tibia. However, no significant difference was found between the anatomical sites and both the overall $(p=0.18)$ and the mechanical $(p=0.17)$ complication rates.

\section{DISCUSSION}

The most important findings of this study were the good functional outcomes achieved in the surviving patients with a relatively low rate of complications in the short-term follow-up. ${ }^{[8]}$ The median MSTS scores of the surviving patients were $88.2 \%$ for upper extremity and $85.3 \%$ for lower extremity. The overall complication rate was $27.2 \%$, and the mechanical complication rate was 22.7\%. Neither of these had a significant relationship with the anatomical location of tumors.

In this study, good functional outcomes were observed in the surviving patients, with a median MSTS score of $86.9 \%$ for the entire cohort at median follow-up of 17 months. Huang et al. ${ }^{[9]}$ studied the outcomes of intercalary prostheses for pathological diaphyseal femoral fractures secondary to metastases and reported a median MSTS score of $84.6 \%$, which is similar to our lower extremity MSTS score of $85.3 \%$. Zhao et al. ${ }^{[10]}$ studied the outcomes of nine patients with pathological humerus fractures secondary to metastatic lesions. They treated the patients with either an intercalary endoprosthesis alone, or an intercalary endoprosthesis combined with a plate. They reported MSTS scores of $81.6 \%$ and $87.3 \%$, respectively, which are comparable to the upper extremity functional outcomes of this study. In a study by Ruggieri et al., ${ }^{[1]}$ outcomes of 24 patients treated with wide resection for primary or metastatic bone tumors involving the diaphysis of the femur, tibia, or humerus and reconstruction using a modular intramedullary diaphyseal segmental defect fixation system (OsteoBridge $^{\mathrm{TM}}$ IDSF [Merete $\mathrm{GmbH}$, Berlin, Germany]) were analyzed at a median follow-up of 29 months. They reported a mean MSTS score of $90 \%$ for upper and $86 \%$ for lower limb, which are comparable to findings of this study. In a recent multi-center study by Benevenia et al., ${ }^{[5]}$ both upper and lower extremity diaphyseal tumors and metastases were treated with a modular intercalary endoprosthesis (OsteoBridge ${ }^{\mathrm{TM}}$ IDSF [Merete $\mathrm{GmbH}$,
Berlin, Germany]). The authors reported an overall MSTS score of $77 \%$, which is slightly lower than our cohort's. Additionally, the authors revealed that resection size was not associated with overall MSTS scores or the sub-scores in their series. In this study, we also did not detect a significant relationship with outcomes and resection length; however, it should be kept in mind that small sample size may increase the type II error rate in our analysis. Although findings of our study are in line with the current literature of equivalent medical device outcomes, it should be interpreted cautiously, since our series include only metastatic lesions, which may affect factors such as patient survival and functional outcomes.

In the present study, the mechanical complication rate of $22.7 \%$ is comparable to that of previous studies with modular intercalary endoprostheses. ${ }^{[5,11,12]}$ In a retrospective study by Friedrich et al., ${ }^{[12]}$ the authors evaluated 15 patients (14 of which were for metastatic tumors) who underwent intercalary reconstruction for diaphyseal bone defects with a modular replacement system at a median follow-up of 24 months. They reported a $20 \%$ rate of revision due to aseptic loosening. Ruggieri et al. ${ }^{[11]}$ reported an implant-related complication rate of $34 \%$, including loosening, rotational instability, breakage, and disassembly of the prostheses. Complications occurred most often at the proximal stem of the implants in femoral reconstructions at two to 24 months after operation and reconstructions for more than $10-\mathrm{cm}$ length of bone resection. In the current study, a higher rate of complications was observed in tibial reconstructions. Due to the low number of patients, we cannot definitively determine the role of anatomical site on implant-related complications. In our opinion, sufficient bone stock after resection and biological milieu of the remaining tissues play vital roles for obtaining durable reconstruction. In the study by Benevenia et al., ${ }^{[5]}$ the mechanical complication rate of $27 \%$ was observed among 41 patients at a median follow-up of 14 months. The authors also reported an association between the type of failure and cement fixation. In their series, cementless fixations had a higher likelihood of resulting in aseptic loosening, whereas cemented ones led to type III failures and complications were mostly observed in the femur (57\%). The lower percentage of aseptic loosening in our cohort might be explained by the low number of cementless cases. The OsteoBridge ${ }^{\mathrm{TM}}$ IDSF (Merete $\mathrm{GmbH}$, Berlin, Germany) implant used in previous studies has a cylindrical clamp at the edges of the intramedullary rods, allowing 
their attachment to the spacer units. The cemented cases in their study failed at the junction between the clamps and rods. The implant used in the current study has been improved by omitting the cylindrical clamp to avoid junctional failure of cemented implants. Nevertheless, breakage of the locking screws that required revision surgery with spacer exchange was also observed in the current study.

Diaphyseal reconstruction with IMN with or without cement augmentation are other available options for the management metastatic diaphyseal lesions. ${ }^{[3]}$ Choice of treatment must be tailored according to general condition and expected survival of the patient. If the expected survival is over 12 months, it is suggested that surgeons should aim for more aggressive treatment options (en bloc resection, endoprostheses) that provide better function and longevity. ${ }^{[3]}$ Compared to IMN for the treatment of long bone diaphyseal metastasis, modular intercalary endoprostheses may provide the benefit of wide resection and reconstruction of the defect. The IMNs mostly achieve fixation with minimal tumor resection, or with wide resection and additional components such as allografts or vascularized autografts which are rarely used in metastatic cancer patients due to the increased risk of delayed union or nonunion. ${ }^{[13]}$ Wide resection has the potential of achieving greater survival in patients with solitary bone metastases, namely in thyroid and renal cell carcinomas. ${ }^{[4,14]}$ Miller et al. ${ }^{[15]}$ found a relationship between IMN revision and underestimation of patient survival, renal cell carcinoma, and radiotherapy-associated fractures. Wedin et al. ${ }^{[16]}$ reported an $8 \%$-failure rate in their series of humeral diaphyseal metastases treated with IMNs. Half of these failures were attributed to nonunion. Intercalary endoprostheses have been also found to be biomechanically superior than IMNs combined with PMMA cement or intercalary allografts with a peak torque of 41.4 $\mathrm{Nm}$ (versus $23.1 \mathrm{Nm}$ and $12.4 \mathrm{Nm}$, respectively). ${ }^{[17]}$ Modular intercalary implants were also found to tolerate higher compressive loads compared to other methods (IMNs, plates, external fixators) in humeral diaphyseal reconstructions. ${ }^{[18]}$ These biomechanical advantages allow the modular intercalary endoprostheses to provide earlier mobilization of patients. To the best of our knowledge, there are no head-to-head studies comparing the clinical results between intercalary endoprostheses and IMN in diaphyseal metastases treatment. Further large-scale, long-term studies are needed to delineate the role of intercalary endoprosthesis in the management of metastatic diaphyseal lesions.

Nonetheless, this study has several limitations. First, the small sample size of our study may increase the type II error in our findings. Second, this study is prone to all inherent limitations of retrospective studies (e.g., recall bias, information bias). A substantial portion of our patients could not be assessed for function due to the relatively high mortality rate in the early postoperative period. This may create bias in functional outcomes and limit us to accurately estimate implant-related complication rates. Finally, this study design also lacks a control group; therefore, we were unable to directly compare our results with different treatment methods, including reconstruction with IMNs.

In conclusion, the good short-term functional results were achieved in surviving patients. Uncomplicated patients were able to perform daily living activities without limitations. The overall rate of complications was relatively low and, among them, mechanical complications were the most commonly encountered problems. Further studies are needed to determine the long-term outcomes of intercalary endoprosthetic management in patients with diaphyseal bone metastases.

\section{Declaration of conflicting interests}

The authors declared no conflicts of interest with respect to the authorship and/or publication of this article.

\section{Funding}

The authors received no financial support for the research and/or authorship of this article.

\section{REFERENCES}

1. Coleman RE. Metastatic bone disease: Clinical features, pathophysiology and treatment strategies. Cancer Treat Rev 2001;27:165-76

2. Mirels H. Metastatic disease in long bones. A proposed scoring system for diagnosing impending pathologic fractures. Clin Orthop Relat Res 1989;(249):256-64.

3. Errani C, Mavrogenis AF, Cevolani L, Spinelli S, Piccioli A, Maccauro G, et al. Treatment for long bone metastases based on a systematic literature review. Eur J Orthop Surg Traumatol 2017;27:205-11.

4. Di Martino A, Martinelli N, Loppini M, Piccioli A, Denaro V. Is endoprosthesis safer than internal fixation for metastatic disease of the proximal femur? A systematic review. Injury 2017;48 Suppl 3:S48-S54.

5. Benevenia J, Kirchner R, Patterson F, Beebe K, Wirtz DC, Rivero $S$, et al. Outcomes of a modular intercalary endoprosthesis as treatment for segmental defects of the femur, tibia, and humerus. Clin Orthop Relat Res 2016;474:539-48.

6. Enneking WF, Dunham W, Gebhardt MC, Malawar M, Pritchard DJ. A system for the functional evaluation of reconstructive procedures after surgical treatment of 
tumors of the musculoskeletal system. Clin Orthop Relat Res 1993;(286):241-6.

7. Henderson ER, O'Connor MI, Ruggieri P, Windhager R, Funovics PT, Gibbons CL, et al. Classification of failure of limb salvage after reconstructive surgery for bone tumours: A modified system Including biological and expandable reconstructions. Bone Joint J 2014;96-B:1436-40.

8. Atik OŞ. What are the expectations of an editor from a scientific article? Jt Dis Relat Surg 2020;31:597-8.

9. Huang HC, Hu YC, Lun DX, Miao J, Wang F, Yang XG, et al. Outcomes of intercalary prosthetic reconstruction for pathological diaphyseal femoral fractures secondary to metastatic tumors. Orthop Surg 2017;9:221-8.

10. Zhao J, Yu XC, Xu M, Zheng $\mathrm{K}, \mathrm{Hu} \mathrm{YC}$, Wang $\mathrm{F}$, et al. Intercalary prosthetic reconstruction for pathologic diaphyseal humeral fractures due to metastatic tumors: Outcomes and improvements. J Shoulder Elbow Surg 2018;27:2013-20.

11. Ruggieri P, Mavrogenis AF, Bianchi G, Sakellariou VI, Mercuri M, Papagelopoulos PJ. Outcome of the intramedullary diaphyseal segmental defect fixation system for bone tumors. J Surg Oncol 2011;104:83-90.

12. Friedrich MJ, Schmolders J, Lob G, Randau TM, Gravius S, Wirtz DC, et al. Intercalary reconstruction for diaphyseal bone defects with a modular replacement system: Clinical results. Oper Orthop Traumatol 2015;27:455-62.

13. Mankin HJ, Gebhardt MC, Jennings LC, Springfield DS, Tomford WW. Long-term results of allograft replacement in the management of bone tumors. Clin Orthop Relat Res 1996;(324):86-97.

14. Fottner A, Szalantzy M, Wirthmann L, Stähler M, BaurMelnyk A, Jansson V, et al. Bone metastases from renal cell carcinoma: Patient survival after surgical treatment. BMC Musculoskelet Disord 2010;11:145.

15. Miller BJ, Soni EE, Gibbs CP, Scarborough MT. Intramedullary nails for long bone metastases: Why do they fail? Orthopedics 2011;34:274.

16. Wedin R, Hansen BH, Laitinen M, Trovik C, Zaikova O, Bergh $\mathrm{P}$, et al. Complications and survival after surgical treatment of 214 metastatic lesions of the humerus. J Shoulder Elbow Surg 2012;21:1049-55.

17. Henry JC, Damron TA, Weiner MM, Higgins ME, Werner FW, Sim FH. Biomechanical analysis of humeral diaphyseal segmental defect fixation. Clin Orthop Relat Res 2002;(396):231-9.

18. Sakellariou VI, Mavrogenis AF, Babis GC, Soucacos PN, Magnissalis EA, Papagelopoulos PJ. Comparison of four reconstructive methods for diaphyseal defects of the humerus after tumor resection. J Appl Biomech 2012;28:568-78. 